\title{
Rate Constants of the Dimerization of PAH Molecules: A Theoretical Study
}

\author{
A.S. Savchenkova1 ${ }^{1}$, A.S. Semenikhin ${ }^{1}$, I.V. Chechet ${ }^{1}$, M. Frenklach ${ }^{2}$, A M. Mebel ${ }^{1,3}$ \\ ${ }^{1}$ Samara National Research University \\ 34, Moskovskoye shosse, Samara, Russia \\ paramonovaanna@mail.ru; cahek-858@yandex.ru; ivanesus@mail.ru \\ ${ }^{2}$ Department of Mechanical Engineering, University of California at Berkeley \\ Berkeley, California, 94720-1740, USA \\ frenklach@berkeley.edu \\ ${ }^{3}$ Department of Chemistry and Biochemistry, Florida International University \\ Miami, FL 33199, USA \\ mebela@fiu.edu
}

\section{Extended Abstract}

The most significant and at the same time poorly studied stage for the formation of soot is the nucleation stage, when the young soot particles are formed from the molecules of the gas phase. Modern concepts of the soot nucleation process consider three possible models, which are based on different interactions of PAHs. There are icospiral mechanism, physical dimerization of PAHs into clusters and dimerization of PAHs by chemical bond. However, it is currently considered that the most likely mechanism for the soot nucleation is the nucleation through the formation of a strong chemical bond.

One of the points of view on the formation of young soot particles is the possibility of dimerization of PAHs in the case when one of the components contains a five-membered ring, and forms strong covalent bonds with another PAH molecule. Frenklach and Mebel in their work [1] showed that one of the ways of such dimerization is the formation of the so-called Ebridge bond, where monomers have a common face of two five-membered rings. This type of dimerization can play an important role in the formation of young soot particles. However, the rate constants of PAH dimerization due to the formation of the E-bridge bond depend, among other things, on the size of the PAHs involved in the process. In this regard, the objective of our research was to determine the dependence of the rate constants for the formation of the E-bridge bond on the size of PAHs involved in the process. To this end, we considered the mechanisms of E-bridge bond formation for three systems: acenaphthalene-naphthalene, aceanthracene-anthracene, and acepyrene-pyrene. The rate constants of its interactions were also determined.

In our studies, geometries of the reactants, products, transition states, and reaction intermediates have been optimized at the DFT B3LYP level of theory. Vibrational frequencies have been computed at the same level of theory to characterize stationary points as local minima or transition states, to obtain zero-point vibrational energy (ZPE) corrections, and for the use in partition function calculations. Temperature- and pressure-dependent rate constants for the reactions considered were evaluated within the framework of RRKM theory in combination with the Master Equation approach (RRKM-ME) [2].

As a result of our work, potential energy surfaces of the formation of the E-bridge bond for the considered systems were built and rate constants were obtained in the form of the sum of modified Arrhenius equations, which are suitable for recording in the Chemkin format and subsequent kinetic modelling.

This work was supported by the grant from the Russian Science Foundation (project No. 19-73-00316).

\section{References}

[1] M. Frenklach and A. M. Mebel, "On the mechanism of soot nucleation”, Phys. Chem. Chem. Phys., vol. 22, pp. 53145331, 2020.

[2] Y. Georgievskii, J. A. Miller, M. P. Burke and S. J. Klippenstein, "Reformulation and Solution of the Master Equation for Multiple-Well Chemical Reactions", J. Phys. Chem. A, vol. 117, pp. 12146-12154, 2013. 